Wisdom Ebong, Alexandra E. Muniz, David R. Khan, Daniel K. Unruh and Jason C. Yarbrough*

\title{
Synthesis and crystal structure of
} nonacarbonyltris[(2-thia-1,3,5-triaza-

\section{7-phosphatricylco[3.3.1.1] decane- ${ }^{1} P$ )- 2,2-dioxide]triruthenium( 0 ) - acetonitrile (7/6), $\mathrm{C}_{25.71} \mathrm{H}_{32.57} \mathrm{~N}_{9.86} \mathrm{O}_{15} \mathrm{P}_{3} \mathrm{~S}_{3} \mathrm{Ru}_{3}$}

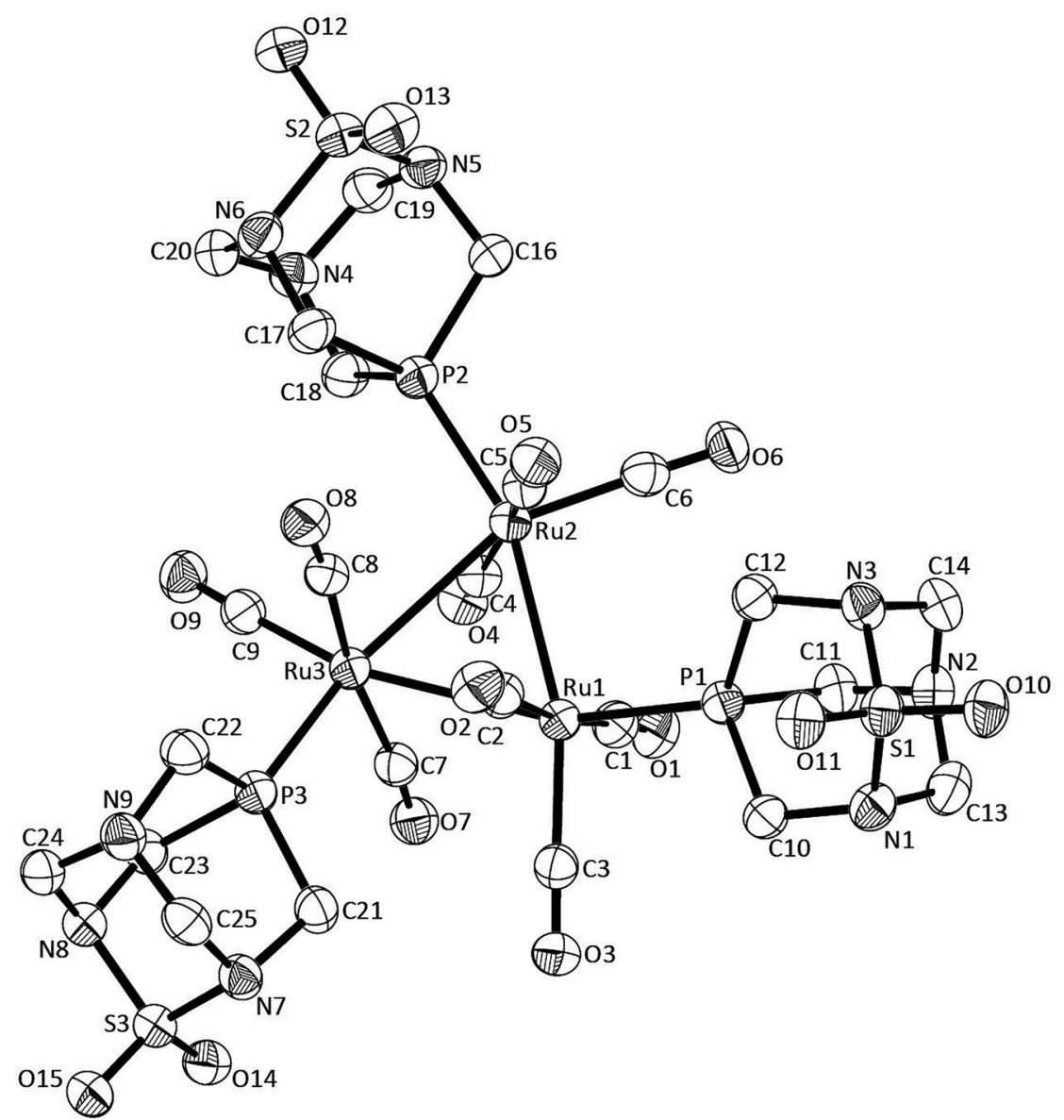

\footnotetext{
*Corresponding author: Jason C. Yarbrough, Department of Chemistry \& Physics, West Texas A\&M University, WTAMU Box 60732, Canyon, TX 79016-0001, USA, E-mail: jyarbrough@wtamu.edu. https://orcid.org/ 0000-0002-1893-2856

Wisdom Ebong, Alexandra E. Muniz and David R. Khan, Department of Chemistry \& Physics, West Texas A\&M University, WTAMU Box 60732, Canyon, TX 79016-0001, USA,

E-mail: wisdomebong@mail.rossmed.edu (W. Ebong), amuniz@wtamu.edu (A.E. Muniz), dkhan@wtamu.edu (D.R. Khan) Daniel K. Unruh, Department of Chemistry \& Biochemistry, Texas Tech University, 1204 Boston Ave., Lubock, TX 79409-1061, USA, E-mail: daniel.unruh@ttu.edu
}

https://doi.org/10.1515/ncrs-2021-0359

Received September 14, 2021; accepted October 22, 2021; published online November 5, 2021

Abstract

$\mathrm{C}_{25.71} \mathrm{H}_{32.57} \mathrm{~N}_{9.86} \mathrm{O}_{15} \mathrm{P}_{3} \mathrm{~S}_{3} \mathrm{Ru}_{3}$, hexagonal, $P 6_{3}$ (no. 173), $a=37.24213(9) \AA, b=37.24213(9) \AA, c=11.64828(3) \AA$, $\beta=90^{\circ}, V=13,991.40(8) \AA^{3}, Z=14, R_{g t}(F)=0.0406$, $w R_{\text {ref }}\left(F^{2}\right)=0.1009, T=100(2) \mathrm{K}$.

CCDC no.: 2117216 
Table 1: Data collection and handling.

\begin{tabular}{ll}
\hline Crystal: & Orange column \\
Size: & $0.18 \times 0.06 \times 0.05 \mathrm{~mm}$ \\
Wavelength: & Cu $K \alpha$ radiation $(1.54178 \AA$ A $)$ \\
$\mu:$ & $12.4 \mathrm{~mm}^{-1}$ \\
Diffractometer, scan mode: & XtaLAB Synergy, $\omega$ \\
$\theta_{\text {max }}$, completeness: & $77.4^{\circ},>99 \%$ \\
$N(h k l)_{\text {measured }}, N(h k l)_{\text {unique }}, R_{\text {int }}:$ & $180,987,19,572,0.049$ \\
Criterion for $I_{\text {obs }}, N(h k l)_{\text {gt }}:$ & $I_{\text {obs }}>2 \sigma\left(I_{\text {obs }}\right), 19,241$ \\
$N(\text { param })_{\text {refined }}:$ & 1254 \\
Programs: & CrysAlis \\
& ABSP [1], SCALE3 \\
& OLEX2 [5] \\
\hline
\end{tabular}

The molecular structure is shown in the figure. Table 1 contains crystallographic data and Table 2 contains the list of the atoms including atomic coordinates and displacement parameters.

\section{Source of material}

The phosphine ligand, 2-thia-1,3,5-triaza-7phosphadamantane-2,2-dioxide $\left(\mathrm{PASO}_{2}\right)$, was prepared according to previously published procedures $[6,7]$. The title compound was obtained by combining $0.360 \mathrm{~g}$ $(0.56 \mathrm{mmol})$ triruthenium dodecacarbonyl with $0.154 \mathrm{~g}$ $(0.743 \mathrm{mmol}) \mathrm{PASO}_{2}$, in $130 \mathrm{ml}$ of xylene. The resulting red solution was refluxed with stirring for $16 \mathrm{~h}$ under argon. The solution was then passed through alumina and the solvent removed in vacuo to yield an orange crystalline powder. Clear orange crystals suitable for X-ray diffraction were obtained via solvent diffusion, where a solution of the title compound in acetonitrile was layered with a two-fold excess of dichloromethane and allowed to sit for four weeks at reduced temperature (approximately $263 \mathrm{~K}$ ).

\section{Experimental details}

Data were collected on a Rigaku XtaLAB Synergy-i Kappa diffractometer equipped with a PhotonJet- $i \mathrm{X}$-ray source operated at $50 \mathrm{~W}(50 \mathrm{kV}, 1 \mathrm{~mA})$ to generate $\mathrm{Cu} \mathrm{K} \alpha$ radiation $(\lambda=1.54178 \AA)$ and a HyPix-6000HE HPC detector. The SHELX-2014 series of programs was used for the solution and refinement of the crystal structure [3, 4]. Hydrogen atoms bound to carbon atoms were geometrically constrained and refined by the riding model. For all $\mathrm{H}$ atoms, $U_{\text {iso }}(\mathrm{H})=1.2 U_{\text {eq }}$ of the carrier atom was assumed (1.5 in the case of methyl groups). The absolute structure was determined by anomalous scattering.
Table 2: Fractional atomic coordinates and isotropic or equivalent isotropic displacement parameters $\left(\AA^{2}\right)$.

\begin{tabular}{|c|c|c|c|c|}
\hline Atom & $x$ & $y$ & $z$ & $U_{\text {iso }}{ }^{*} / U_{\text {eq }}$ \\
\hline Ru1 & $0.27257(2)$ & $0.19371(2)$ & $0.70735(4)$ & $0.03148(10)$ \\
\hline Ru2 & $18429(2)$ & $15030(2)$ & $0.70543(4)$ & 0.0321 \\
\hline Ru3 & $0.22916(2)$ & $0.23868(2)$ & $0.68830(4)$ & $0.03202(10)$ \\
\hline Ru4 & $0.47266(2)$ & $0.57221(2)$ & $0.50526(4)$ & 0.033 \\
\hline Ru5 & $0.51829(2)$ & $0.65985(2)$ & $0.53670(4)$ & $0.03386(10)$ \\
\hline Ru6 & $0.56126(2)$ & $0.61429(2)$ & $0.51118(4)$ & $0.03300(10)$ \\
\hline Ru7 & $0.62640(2)$ & $0.34074(2)$ & $0.51535(5)$ & 0.0402 \\
\hline N7 & $0.36082(15)$ & $0.36315(15)$ & $0.5815(5)$ & $0.0366(10)$ \\
\hline S1 & $0.33708(5)$ & $0.10820(5)$ & 0.49979 (14) & $0.0387(3)$ \\
\hline S2 & $0.02883(4)$ & $0.09322(4)$ & 0.52727 (13) & .0368 (3) \\
\hline S3 & $0.35897(4)$ & $0.39835(4)$ & 0.66 & 0.0353 \\
\hline S4 & 0.31704 (4) & $0.52069(4)$ & $0.67086(14)$ & $0.0382(3)$ \\
\hline S5 & $0.64719(4)$ & $0.82043(4)$ & $0.54765(13)$ & $0.0358(3)$ \\
\hline S6 & $2(5)$ & $0.51739(5)$ & 0.68897 (15) & $0.0423(3)$ \\
\hline S7 & $0.47674(5)$ & $0.25030(5)$ & $0.71995(15)$ & .0434 (3) \\
\hline P1 & & & & (3) \\
\hline P2 & $4(4)$ & $0.13591(4)$ & (13) & $0(3)$ \\
\hline P3 & $3(4)$ & $3(4)$ & 0.6 & $6(3)$ \\
\hline P4 & $5(4)$ & $0(4)$ & (13) & $8(3)$ \\
\hline P5 & $0.56906(4)$ & $0.72507(4)$ & 0.58 & .0347 (3) \\
\hline P6 & $9(4)$ & $8(4)$ & 13) & $40(3)$ \\
\hline P7 & (5) & $6(5)$ & (15) & $2(3)$ \\
\hline 01 & (15) & 0.16 & $5(4)$ & (10) \\
\hline 02 & (15) & 0.2 & $(4)$ & (10) \\
\hline 03 & $0.35954(14)$ & 0.25 & $2(4)$ & $.0428(9)$ \\
\hline 04 & $2(14)$ & 0.17 & $(4)$ & 07 (9) \\
\hline 05 & 15) & 0.1 & $(4)$ & (10) \\
\hline 06 & & & & \\
\hline 07 & 14) & 0.2 & $(4)$ & $7(9)$ \\
\hline 08 & 0.19 & 0.2 & $5(4)$ & $0(9)$ \\
\hline N8 & 5) & 0.3 & & (10) \\
\hline 09 & $33(14)$ & 0.2 & $(5)$ & (10) \\
\hline N9 & 34 (17) & 0.36 & (5) & (11) \\
\hline 010 & & 0.0 & & $0.0452(10)$ \\
\hline N1 & & 0.5 & (5) & (10) \\
\hline 01 & 6) & 0.1 & & (10) \\
\hline N11 & & 0.5 & $3(5)$ & 0.03 \\
\hline 012 & 3) & 0.0 & $5(4)$ & $8(9)$ \\
\hline N12 & & & & 0.03 \\
\hline 01 & & & & \\
\hline N13 & & 0.781 & & $0.0361(10$ \\
\hline 014 & 0.36307 (14) & 0.38 & $0.7812(4)$ & $12(9$ \\
\hline N14 & $0.65164(15)$ & 0.78 & $0.6247(5)$ & $0.0360(10)$ \\
\hline 015 & & $0.43817(13)$ & & $0.0427(10$ \\
\hline C15 & 8) & 0.51 & $9(6)$ & $3(12$ \\
\hline N15 & 0.59927 (15) & $0.81047(15)$ & $0.5761(5)$ & $0.0356(10$ \\
\hline N1 & $0.35826(17)$ & $0.13400(17)$ & $0.6207(5)$ & $0.0394(10$ \\
\hline N2 & 0.30019 (18) & $0.07891(17)$ & $0.7355(5)$ & $0.0414(11$ \\
\hline N3 & & $0.08215(15)$ & & $0.0391(10)$ \\
\hline N4 & $0.04855(16)$ & $0.13782(16)$ & $0.7538(5)$ & $0.0378(10)$ \\
\hline N5 & $0.03428(15)$ & $0.07410(16)$ & $0.6489(5)$ & $0.0366(1 c$ \\
\hline N6 & $0.05885(16)$ & $0.14406(16)$ & $0.5431(5)$ & $0.0379(10$ \\
\hline $\mathrm{C} 1$ & $0.25802(18)$ & 0.17329 (19) & $0.8636(6)$ & $0.0377(12$ \\
\hline C2 & $0.27899(18)$ & $0.20916(18)$ & $0.5467(6)$ & $0.0367(12$ \\
\hline C3 & $0.32728(18)$ & $0.23556(17)$ & $0.7478(5)$ & 0.0346 \\
\hline
\end{tabular}


Table 2: (continued)

\begin{tabular}{|c|c|c|c|}
\hline Atom & $x$ & $y$ & $z$ \\
\hline C4 & $0.18108(17)$ & 0.16907 (17) & $0.8591(6)$ \\
\hline C5 & $0.19451(18)$ & $0.14229(18)$ & $0.5465(6)$ \\
\hline C6 & $0.17424(18)$ & 0.09692 (19) & $0.7458(6)$ \\
\hline $\mathrm{C} 7$ & $0.25228(17)$ & $0.24765(17)$ & $0.8422(6)$ \\
\hline C8 & $0.21010(17)$ & $0.22416(17)$ & $0.5311(6)$ \\
\hline C9 & $0.18674(18)$ & $0.25058(18)$ & $0.7296(6)$ \\
\hline $\mathrm{C} 10$ & $0.34634(18)$ & 0.16589 (18) & $0.6429(6)$ \\
\hline $\mathrm{H} 10 \mathrm{~A}$ & 0.354615 & 0.184822 & 0.576081 \\
\hline $\mathrm{H} 10 \mathrm{~B}$ & 0.361912 & 0.182489 & 0.710462 \\
\hline $\mathrm{C} 11$ & $0.2823(2)$ & 0.10433 (19) & $0.7754(6)$ \\
\hline $\mathrm{H} 11 \mathrm{~A}$ & 0.295556 & 0.117927 & 0.848744 \\
\hline H11B & 0.252295 & 0.086147 & 0.789555 \\
\hline C12 & $0.26837(18)$ & $0.10940(18)$ & $0.5430(6)$ \\
\hline $\mathrm{H} 12 \mathrm{~A}$ & 0.237971 & 0.091907 & 0.552189 \\
\hline $\mathrm{H} 12 \mathrm{~B}$ & 0.273713 & 0.12639 & 0.472978 \\
\hline $\mathrm{C} 13$ & $0.3450(2)$ & $0.1033(2)$ & $0.7185(6)$ \\
\hline $\mathrm{H} 13 \mathrm{~A}$ & 0.355636 & 0.084105 & 0.704025 \\
\hline H13B & 0.357939 & 0.118572 & 0.790216 \\
\hline C14 & $0.2801(2)$ & 0.05580 (19) & $0.6326(6)$ \\
\hline $\mathrm{H} 14 \mathrm{~A}$ & 0.249887 & 0.038977 & 0.647192 \\
\hline H14B & 0.290339 & 0.036429 & 0.616038 \\
\hline C16 & $0.07695(17)$ & 0.08037 (18) & $0.6626(6)$ \\
\hline $\mathrm{H} 16 \mathrm{~A}$ & 0.083383 & 0.068196 & 0.595636 \\
\hline H16B & 0.077722 & 0.065471 & 0.732092 \\
\hline N16 & $0.56799(17)$ & $0.49485(16)$ & $0.6628(5)$ \\
\hline 016 & $0.48419(16)$ & $0.55426(16)$ & $0.7565(5)$ \\
\hline $\mathrm{C} 17$ & $0.10391(18)$ & 0.15685 (19) & $0.5479(6)$ \\
\hline H17A & 0.120629 & 0.187498 & 0.549311 \\
\hline H17B & 0.111331 & 0.147125 & 0.477531 \\
\hline N17 & $0.57932(17)$ & $0.49425(16)$ & $0.4535(5)$ \\
\hline 017 & 0.46609 (14) & $0.59873(14)$ & $0.2596(4)$ \\
\hline C18 & 0.09169 (18) & 0.15113 (19) & $0.7848(6)$ \\
\hline $\mathrm{H} 18 \mathrm{~A}$ & 0.092137 & 0.138588 & 0.859339 \\
\hline $\mathrm{H} 18 \mathrm{~B}$ & 0.107325 & 0.181639 & 0.793953 \\
\hline N18 & $0.63968(16)$ & 0.54398 (17) & 0.5705 (5) \\
\hline 018 & $0.45614(15)$ & $0.48648(14)$ & $0.4373(5)$ \\
\hline C19 & $0.02301(18)$ & 0.09283 (19) & $0.7474(6)$ \\
\hline H19A & -0.0064 & 0.085324 & 0.73951 \\
\hline $\mathrm{H} 19 \mathrm{~B}$ & 0.025808 & 0.08071 & 0.820149 \\
\hline N19 & $0.49880(18)$ & $0.22396(18)$ & $0.6723(6)$ \\
\hline 019 & $0.49182(16)$ & 0.64037 (15) & 0.7889 (5) \\
\hline $\mathrm{C} 20$ & 0.04550 (19) & 0.15728 (19) & $0.6506(6)$ \\
\hline $\mathrm{H} 20 \mathrm{~A}$ & 0.06288 & 0.187713 & 0.659123 \\
\hline $\mathrm{H} 20 \mathrm{~B}$ & 0.016414 & 0.150677 & 0.640978 \\
\hline $\mathrm{N} 20$ & $0.48096(19)$ & 0.28078 (19) & $0.6111(5)$ \\
\hline 020 & 0.44937 (14) & $0.68014(14)$ & $0.5063(5)$ \\
\hline C21 & $0.33455(18)$ & 0.32017 (18) & $0.6278(6)$ \\
\hline $\mathrm{H} 21 \mathrm{~A}$ & 0.343365 & 0.319103 & 0.707353 \\
\hline $\mathrm{H} 21 \mathrm{~B}$ & 0.338978 & 0.30057 & 0.580859 \\
\hline $\mathrm{N} 21$ & $0.4819(2)$ & $0.2307(2)$ & $0.4711(6)$ \\
\hline 021 & $0.57471(16)$ & $0.63320(18)$ & $0.7701(5)$ \\
\hline $\mathrm{C} 22$ & $0.2752(2)$ & 0.31776 (19) & 0.4777 (5) \\
\hline $\mathrm{H} 22 \mathrm{~A}$ & 0.278187 & 0.298518 & 0.425088 \\
\hline $\mathrm{H} 22 \mathrm{~B}$ & 0.247543 & 0.31467 & 0.464661 \\
\hline $\mathrm{N} 22$ & $0.3148(2)$ & $0.2842(2)$ & $0.2374(6)$ \\
\hline 022 & $0.54347(16)$ & 0.58749 (16) & 0.2585 (4) \\
\hline
\end{tabular}

Table 2: (continued)

\begin{tabular}{|c|c|c|c|c|}
\hline Atom & $x$ & $y$ & $z$ & $U_{\text {iso }} * / U_{\text {eq }}$ \\
\hline $\mathrm{C} 23$ & $0.28010(17)$ & $0.34877(17)$ & $0.6982(6)$ & $0.0361(11)$ \\
\hline $\mathrm{H} 23 \mathrm{~A}$ & 0.252182 & 0.345766 & 0.693561 & $0.043^{*}$ \\
\hline $\mathrm{H} 23 \mathrm{~B}$ & 0.286866 & 0.348841 & 0.780387 & $0.043^{*}$ \\
\hline N23 & $0.6067(2)$ & $0.7132(3)$ & $0.9833(7)$ & $0.0642(18)$ \\
\hline 023 & $0.64966(14)$ & $0.67933(14)$ & $0.4492(5)$ & $0.0468(11)$ \\
\hline $\mathrm{C} 24$ & $0.30272(19)$ & 0.39094 (18) & $0.5204(6)$ & $0.0404(12)$ \\
\hline $\mathrm{H} 24 \mathrm{~A}$ & 0.274217 & 0.386058 & 0.510551 & 0.049 * \\
\hline $\mathrm{H} 24 \mathrm{~B}$ & 0.32216 & 0.419109 & 0.491245 & $0.049^{*}$ \\
\hline 024 & $0.33143(14)$ & 0.50539 (14) & $0.7607(5)$ & $0.0447(10)$ \\
\hline $\mathrm{C} 25$ & $0.3488(2)$ & 0.36703 (19) & $0.4603(6)$ & $0.0404(13)$ \\
\hline $\mathrm{H} 25 \mathrm{~A}$ & 0.368705 & 0.395015 & 0.430817 & $0.049 *$ \\
\hline $\mathrm{H} 25 \mathrm{~B}$ & 0.35109 & 0.346545 & 0.411148 & 0.049 * \\
\hline 025 & $0.27592(14)$ & $0.51410(14)$ & $0.6731(5)$ & $0.0444(10)$ \\
\hline $\mathrm{C} 26$ & $0.36450(17)$ & $0.50675(17)$ & $0.5360(6)$ & $0.0371(12)$ \\
\hline $\mathrm{H} 26 \mathrm{~A}$ & 0.365388 & 0.492552 & 0.46516 & $0.044^{\star}$ \\
\hline $\mathrm{H} 26 \mathrm{~B}$ & 0.369237 & 0.492837 & 0.601934 & $0.044^{*}$ \\
\hline 026 & $0.67483(14)$ & $0.86039(14)$ & $0.5943(4)$ & $0.0423(10)$ \\
\hline $\mathrm{C} 27$ & $0.38406(18)$ & 0.57976 (19) & $0.4195(6)$ & $0.0371(12)$ \\
\hline $\mathrm{H} 27 \mathrm{~A}$ & 0.400928 & 0.610313 & 0.413054 & $0.045^{*}$ \\
\hline $\mathrm{H} 27 \mathrm{~B}$ & 0.384824 & 0.56772 & 0.344382 & $0.045^{\star}$ \\
\hline 027 & 0.64927 (14) & $0.81242(14)$ & $0.4294(4)$ & $0.0412(9)$ \\
\hline $\mathrm{C} 28$ & $0.39348(18)$ & 0.58203 (19) & $0.6590(6)$ & 0.0377 (12) \\
\hline $\mathrm{H} 28 \mathrm{~A}$ & 0.399018 & 0.57053 & 0.729041 & $0.045^{\star}$ \\
\hline $\mathrm{H} 28 \mathrm{~B}$ & 0.411509 & 0.612549 & 0.661263 & $0.045^{*}$ \\
\hline 028 & $0.62811(16)$ & $0.54566(17)$ & $0.7812(5)$ & $0.0509(11)$ \\
\hline C29 & $0.33803(18)$ & 0.58714 (19) & $0.5535(6)$ & $0.0398(12)$ \\
\hline $\mathrm{H} 29 \mathrm{~A}$ & 0.309386 & 0.582032 & 0.561447 & $0.048^{*}$ \\
\hline $\mathrm{H} 29 \mathrm{~B}$ & 0.356808 & 0.617488 & 0.54812 & $0.048^{*}$ \\
\hline 029 & $0.62862(15)$ & $0.48528(16)$ & $0.6959(5)$ & $0.0497(11)$ \\
\hline C30 & 0.31369 (19) & $0.52328(19)$ & $0.4503(6)$ & $0.0414(13)$ \\
\hline $\mathrm{H} 30 \mathrm{~A}$ & 0.316224 & 0.511381 & 0.376773 & $0.05^{*}$ \\
\hline $\mathrm{H} 30 \mathrm{~B}$ & 0.284688 & 0.517229 & 0.456907 & $0.05^{\star}$ \\
\hline 030 & $0.61432(18)$ & $0.32376(19)$ & $0.2565(5)$ & $0.0546(12)$ \\
\hline C 31 & 0.56841 (19) & $0.73870(18)$ & $0.7406(6)$ & 0.0377 (12) \\
\hline $\mathrm{H} 31 \mathrm{~A}$ & 0.540842 & 0.735077 & 0.759073 & $0.045^{\star}$ \\
\hline H31B & 0.572716 & 0.719524 & 0.789863 & $0.045^{\star}$ \\
\hline 031 & $0.43394(16)$ & $0.22140(17)$ & $0.7355(5)$ & $0.0514(11)$ \\
\hline C 32 & $0.56791(17)$ & $0.76985(17)$ & $0.5255(6)$ & $0.0358(11)$ \\
\hline $\mathrm{H} 32 \mathrm{~A}$ & 0.572933 & 0.77034 & 0.441937 & $0.043^{*}$ \\
\hline H32B & 0.539925 & 0.766235 & 0.536395 & $0.043^{*}$ \\
\hline 032 & $0.50132(15)$ & $0.27560(16)$ & $0.8122(5)$ & $0.0491(11)$ \\
\hline C33 & $0.62504(18)$ & 0.74269 (17) & $0.5787(6)$ & $0.0361(11)$ \\
\hline H33A & 0.630856 & 0.723195 & 0.621538 & $0.043^{*}$ \\
\hline H33B & 0.632231 & 0.742082 & 0.497172 & $0.043^{*}$ \\
\hline 033 & $0.55090(14)$ & $0.67887(14)$ & $0.2879(4)$ & $0.0434(10)$ \\
\hline C34 & $0.47037(17)$ & 0.59034 (18) & $0.3503(6)$ & $0.0363(12)$ \\
\hline 034 & $0.63399(17)$ & 0.35524 (18) & $0.7762(5)$ & $0.0534(12)$ \\
\hline C35 & 0.48153 (19) & 0.56259 (19) & $0.6637(6)$ & $0.0388(12)$ \\
\hline 035 & $0.6139(2)$ & 0.41419 (19) & $0.4726(6)$ & $0.0611(14)$ \\
\hline C36 & $0.50170(18)$ & $0.64539(18)$ & $0.6950(6)$ & $0.0403(13)$ \\
\hline C37 & $0.53883(18)$ & $0.66992(17)$ & $0.3796(6)$ & $0.0357(11)$ \\
\hline C38 & $0.47575(18)$ & $0.67301(17)$ & $0.5146(6)$ & $0.0380(12)$ \\
\hline C39 & $0.56763(19)$ & $0.6267(2)$ & $0.6738(6)$ & 0.0405 (13) \\
\hline $\mathrm{C} 40$ & $0.61645(18)$ & $0.65579(18)$ & $0.4754(6)$ & $0.0380(12)$ \\
\hline C41 & $0.54788(19)$ & 0.59789 (19) & $0.3517(6)$ & $0.0381(12)$ \\
\hline C42 & $0.59366(18)$ & $0.81126(18)$ & $0.7045(6)$ & $0.0373(11)$ \\
\hline
\end{tabular}


Table 2: (continued)

\begin{tabular}{|c|c|c|c|c|}
\hline Atom & $x$ & $y$ & $z$ & $U_{\text {iso }} * / U_{\text {eq }}$ \\
\hline $\mathrm{H} 42 \mathrm{~A}$ & 0.613128 & 0.839441 & 0.73347 & $0.045^{\star}$ \\
\hline $\mathrm{H} 42 \mathrm{~B}$ & 0.565141 & 0.805524 & 0.720238 & $0.045^{\star}$ \\
\hline $\mathrm{C} 43$ & 0.64185 (19) & $0.7885(2)$ & $0.7492(6)$ & $0.0389(12)$ \\
\hline $\mathrm{H} 43 \mathrm{~A}$ & 0.645177 & 0.76788 & 0.794712 & $0.047^{\star}$ \\
\hline H43B & 0.662131 & 0.81637 & 0.778234 & $0.047^{*}$ \\
\hline C44 & 0.55298 (19) & $0.5254(2)$ & $0.6563(6)$ & $0.0396(12)$ \\
\hline $\mathrm{H} 44 \mathrm{~A}$ & 0.559936 & 0.541379 & 0.728809 & $0.048^{*}$ \\
\hline H44B & 0.522422 & 0.510181 & 0.648191 & $0.048^{*}$ \\
\hline C45 & $0.5648(2)$ & $0.52326(19)$ & $0.4207(6)$ & $0.0388(12)$ \\
\hline $\mathrm{H} 45 \mathrm{~A}$ & 0.534604 & 0.50747 & 0.406348 & $0.047^{\star}$ \\
\hline H45B & 0.578716 & 0.537646 & 0.348742 & $0.047^{\star}$ \\
\hline C46 & $0.63153(19)$ & 0.57915 (19) & $0.5563(6)$ & $0.0385(12)$ \\
\hline $\mathrm{H} 46 \mathrm{~A}$ & 0.64754 & 0.59611 & 0.489671 & $0.046^{*}$ \\
\hline $\mathrm{H} 46 \mathrm{~B}$ & 0.64153 & 0.596975 & 0.6254 & $0.046^{*}$ \\
\hline C47 & $0.5583(2)$ & $0.4702(2)$ & $0.5539(7)$ & $0.0436(13)$ \\
\hline $\mathrm{H} 47 \mathrm{~A}$ & 0.565905 & 0.448407 & 0.56416 & $0.052^{\star}$ \\
\hline H47B & 0.528049 & 0.456121 & 0.540311 & $0.052^{*}$ \\
\hline $\mathrm{C} 48$ & $0.6241(2)$ & $0.5156(2)$ & $0.4680(6)$ & $0.0423(13)$ \\
\hline $\mathrm{H} 48 \mathrm{~A}$ & 0.637293 & 0.531996 & 0.397995 & $0.051^{*}$ \\
\hline $\mathrm{H} 48 \mathrm{~B}$ & 0.632884 & 0.494598 & 0.475527 & $0.051^{*}$ \\
\hline C49 & $0.6333(2)$ & $0.3499(2)$ & $0.6797(6)$ & $0.0433(13)$ \\
\hline $\mathrm{C} 50$ & $0.6207(2)$ & $0.3293(2)$ & $0.3515(6)$ & 0.0459 (15) \\
\hline C51 & $0.6194(2)$ & $0.3872(2)$ & $0.4911(7)$ & 0.0445 (14) \\
\hline C52 & $0.5255(2)$ & $0.3132(2)$ & $0.5918(7)$ & 0.0445 (14) \\
\hline $\mathrm{H} 52 \mathrm{~A}$ & 0.536666 & 0.329266 & 0.663462 & $0.053^{*}$ \\
\hline H52B & 0.526729 & 0.332516 & 0.531593 & $0.053^{*}$ \\
\hline C53 & $0.5444(2)$ & $0.2509(2)$ & $0.6595(6)$ & 0.0440 (13) \\
\hline $\mathrm{H} 53 \mathrm{~A}$ & 0.556654 & 0.233522 & 0.638964 & $0.053^{*}$ \\
\hline H53B & 0.556368 & 0.264384 & 0.734037 & $0.053^{*}$ \\
\hline C54 & $0.5253(2)$ & $0.2574(2)$ & $0.4332(6)$ & 0.0477 (15) \\
\hline H54A & 0.525795 & 0.274568 & 0.367646 & $0.057^{*}$ \\
\hline H54B & 0.536389 & 0.239662 & 0.406266 & $0.057^{*}$ \\
\hline $\mathrm{C} 55$ & $0.4630(2)$ & $0.2551(3)$ & $0.5049(7)$ & $0.0513(15)$ \\
\hline $\mathrm{H} 55 \mathrm{~A}$ & 0.433048 & 0.236034 & 0.517422 & $0.062^{\star}$ \\
\hline H55B & 0.46592 & 0.273716 & 0.440629 & $0.062^{*}$ \\
\hline C56 & $0.4789(2)$ & $0.2027(2)$ & $0.5600(8)$ & $0.0522(16)$ \\
\hline $\mathrm{H} 56 \mathrm{~A}$ & 0.492303 & 0.187044 & 0.533043 & $0.063^{*}$ \\
\hline H56B & 0.449281 & 0.182569 & 0.573955 & $0.063^{*}$ \\
\hline $\mathrm{C} 57$ & $0.2895(2)$ & $0.2510(2)$ & $0.2216(6)$ & $0.0430(13)$ \\
\hline C58 & $0.2568(2)$ & $0.2081(2)$ & $0.2022(7)$ & 0.0474 (14) \\
\hline $\mathrm{H} 58 \mathrm{~A}$ & 0.244078 & 0.205923 & 0.126897 & $0.071^{*}$ \\
\hline $\mathrm{H} 58 \mathrm{~B}$ & 0.235646 & 0.199805 & 0.262149 & $0.071^{*}$ \\
\hline $\mathrm{H} 58 \mathrm{C}$ & 0.268747 & 0.189928 & 0.204394 & $0.071^{*}$ \\
\hline C59 & $0.5798(3)$ & $0.6804(3)$ & $1.0045(7)$ & 0.0578 (19) \\
\hline $\mathrm{C} 60$ & $0.5465(3)$ & $0.6399(3)$ & $1.0295(7)$ & $0.0602(19)$ \\
\hline $\mathrm{H} 60 \mathrm{~A}$ & 0.528563 & 0.628758 & 0.96206 & $0.09^{\star}$ \\
\hline $\mathrm{H} 60 \mathrm{~B}$ & 0.530438 & 0.641339 & 1.094207 & $0.09^{*}$ \\
\hline $\mathrm{H} 60 \mathrm{C}$ & 0.557672 & 0.621851 & 1.049642 & $0.09 *$ \\
\hline
\end{tabular}

\section{Comment}

The structural chemistry of metal-carbonyl cluster compounds and their derivatives has been an area of significant interest for many decades. This stems from their application in a variety of endeavors, including heterogeneous catalyst design as well as materials chemistry and engineering research [8]. Indeed, many structural studies are reported in the literature and not surprisingly therefore, standard empirical rules have been established which make the geometry and stoichiometry of ligand binding reasonably predictable in such compounds [9-12]. Herein we report the synthesis and crystal structure of a solvated, triangulo-triruthenium compound, tri-substituted with the small, cage-like phosphine ligand, 2-thia-1,3,5-triaza7-phosphaadamantane 2,2-dioxide $\quad\left(\mathrm{PASO}_{2}\right)$, $\mathrm{Ru}_{3}(\mathrm{CO})_{9}\left(\mathrm{PASO}_{2}\right)_{3}: 6 / 7 \mathrm{C}_{2} \mathrm{H}_{3} \mathrm{~N}$. The synthesis of the title compound, $\mathrm{Ru}_{3}(\mathrm{CO})_{9}\left(\mathrm{PASO}_{2}\right)_{3}$, was accomplished by the thermal reaction of $\mathrm{Ru}_{3}(\mathrm{CO})_{12}$ and the $\mathrm{PASO}_{2}$ phosphine. Employing the crystallization method of solvent diffusion, the compound was found to crystallize into the noncentrosymmetric, hexagonal space group, $P 6_{3}$. The asymmetric unit consists of 2 and $1 / 3$ triruthenium clusters and two molecules of acetonitrile solvent. Further, each complex was determined to consist of a triangular $\mathrm{Ru}_{3}$ core with interior bond angles of approximately $60^{\circ}$, ranginging from 59.664(13) to $60.367(13)^{\circ}$ for the two crystallographically distinct $\mathrm{Ru}_{3}$ cores in the asymmetric unit. Additionally, the corresponding $\mathrm{Ru}-\mathrm{Ru}$ bond distances were all of similar length, ranging from 2.8473(5) to 2.8710(6) $\AA$, consistent with the observed $\mathrm{Ru}-\mathrm{Ru}$ bond lengths in the parent compound, $\mathrm{Ru}_{3}(\mathrm{CO})_{12}$ which was previously reported in reference [13]. Furthermore, the crystallographically imposed bond angles and lengths corresponding to $\mathrm{Ru} 7$ are $60.0^{\circ}$ and 2.8661(8) $\AA$ respectively. Analogous $\mathrm{Ru}_{3}(\mathrm{CO})_{9}\left(\mathrm{PR}_{3}\right)_{3}$ compounds reported previously [14-16] show similar bond lengths and angles with respect to the $\mathrm{Ru}_{3}$ core. A thermal ellipsoid depiction of the title compound is shown in the figure. Each ruthenium bears three terminal carbonyl ligands and an equatorially positioned $\mathrm{PASO}_{2}$ ligand, providing a somewhat distorted octahedral environment about each Ru center. The ruthenium-phosphorus bond lengths Ru1-P1, Ru2-P2, Ru3-P3, Ru4-P4, Ru5-P5, Ru6-P6 and Ru7-P7 are all fairly consistent and range from 2.2874(14) to 2.3042(14) $\AA$. In all instances, the equatorial $\mathrm{Ru}-\mathrm{CO}$ bond distances are shorter than those in the axial positions. These observations are consistent with the most analogous compound found in the chemical literature, $\mathrm{Ru}_{3}(\mathrm{CO})_{9}(\mathrm{PTA})_{3}[15]$. Additionally, and as expected with binding of the $\mathrm{PASO}_{2}$ phosphine, the equatorial $\mathrm{Ru}-\mathrm{CO}$ bond lengths are shorter than those in the structure of the unsubstituted parent compound, $\mathrm{Ru}_{3}(\mathrm{CO})_{12}$ [13]. On this point, we can further note that binding of the phosphine ligands brings with it steric interactions which introduce significant distortion of the molecular symmetry from the $D_{3 h}$ molecular symmetry of $\mathrm{Ru}_{3}(\mathrm{CO})_{12}$ to $D_{3}$ symmetry for the title 
compound. This is readily observed in the $\mathrm{Ru}-\mathrm{Ru}-\mathrm{CO}_{a x}$ bond angles of the axial ligands, the average value of which is $78.6(9)^{\circ}$ in contrast to that of roughly $90^{\circ}$ for the parent compound, $\mathrm{Ru}_{3}(\mathrm{CO})_{12}[13]$.

Author contributions: All the authors have accepted responsibility for the entire content of this submitted manuscript and approved submission.

Research funding: Funding was generously provided by the Welch Foundation (grant number AE-0025), the Killgore Research Grant program and the Ross Wilson Endowed Chair in Chemistry (Jason C. Yarbrough) at West Texas A\&M University.

Conflict of interest statement: The authors declare no conflicts of interest regarding this article.

\section{References}

1. Agilent Technologies Ltd. CrysAlis ${ }^{\text {Pro }}$; Yarntton, Oxfordshire, England, 2018.

2. Oxford Diffraction Ltd. SCALE 3 ABSPACK: Abingdon, Oxfordshire, England, 2005.

3. Sheldrick G. M. Crystal structure refinement with SHELXL. Acta Crystallogr. 2015, C71, 3-8.

4. Sheldrick G. M. SHELXT - inetgrated space-group and crystalstructure determination. Acta Crystallogr. 2015, A7, 3-8.

5. Dolomanov 0. V., Bourhis L. J., Gildea R. J., Howard J. A. K., Puschmann H. OLEX2: a complete structure solution, refinement and analysis program. J. Appl. Crystallogr. 2009, 42, 339-341.

6. Darensbourg D. J., Yarbrough J. C., Lewis S. J. 2-Thia1,3,5-triazaphosphaadamantane 2,2-dioxide $\left(\mathrm{PASO}_{2}\right)$. Comparative structural and reactivity investigation with the water-soluble phosphine ligand 1,3,5-triazaphosphaadamantane (PTA). Organometallics 2003, 22, 2050-2056.

7. Daigle D. J., Pepperman A. B., Boudreaux G. Phosphaadamantanes synthesis of 2-thia-1,3,5-triazaphosphaadamantane 2,2-dioxide and derivatives. J. Heterocycl. Chem. 1974, 11, 1085-1086.

8. Lou Z., Castleman A. W., Khanna S. N. Reactivity of metal clusters. Chem. Rev. 2016, 116, 14456-14492.

9. Lauher J. W. A surface force field model for the molecular mechanics simulation of ligand structures in transition-metal carbonyl clusters. J. Am. Chem. Soc. 1986, 108, 1521-1531.

10. Lauher J. W. The bonding capabilities of transition metal clusters. IV. Three classes of carbonyl cluster compounds. J. Organomet. Chem. 1981, 213, 25-34.

11. Lauher J. W. Bonding capabilities of transition metal clusters. 2. Relationships to bulk metals. J. Am. Chem. Soc. 1979, 101, 2604-2607.

12. Lauher J. W. The bonding capabilities of transition metal clusters. J. Am. Chem. Soc. 1978, 100, 5305-5315.

13. Churchill M. R., Hollander F. J., Hutchinson J. P. An accurate redetermination of the structure of triruthenium dodecacarbonyl, $\mathrm{Ru}_{3}(\mathrm{CO})_{12}$. Inorg. Chem. 1977, 16, 2655-2659.

14. Arce A. J., De Sanctis Y., Goite M. C., Machado R., Otero Y., Gonzalez T. Insertion of a phospholide unit into a metal-metal bond: synthesis and $\mathrm{x}$-ray structure of $\left[\mathrm{Ru}_{3}(\mathrm{CO})_{9}(\mu\right.$ : $\left.\left.\eta^{1}: \eta^{5}-\mathrm{PC}_{4} \mathrm{H}_{2} \mathrm{Me}_{2}\right)\left(\eta^{1}-\mathrm{C}_{6} \mathrm{H}_{5}\right)\right]$. Inorg. Chim. Acta 2012, 392, 241-245.

15. Darensbourg D. J., Beckford F. A., Reibenspies J. H. Water-soluble organometallic compounds. 8. Synthesis, spectral properties and crystal structures of 1,3,5-triaza-7-phosphaadamantane (PTA) derivatives of metal carbonyl clusters: $\mathrm{Ru}_{3}(\mathrm{CO})_{9}(\mathrm{PTA})_{3}$ and $\mathrm{Ir}_{4}(\mathrm{CO})_{7}(\mathrm{PTA})_{5}$. J. Cluster Sci. 2000, 11, 95-107.

16. Bruce M. I., Liddell M. J., Shawkataly O. LVII. Stereochemistry of group 15 ligand-substituted derivatives of $\mathrm{M}_{3}(\mathrm{CO})_{12}(\mathrm{M}=\mathrm{Ru}$, Os). C. X-ray structures of $\mathrm{Ru}_{3}(\mathrm{CO})_{9}(\mathrm{~L})_{3}\left(\mathrm{~L}=\mathrm{PMe}_{2}\left(\mathrm{CH}_{2} \mathrm{Ph}\right)\right.$, $\mathrm{PMe}_{2} \mathrm{Ph}, \mathrm{AsMe}_{2} \mathrm{Ph}, \mathrm{PPh}(\mathrm{OMe})_{2}, \mathrm{P}(\mathrm{OEt})_{3}$, and $\left.\mathrm{P}\left(\mathrm{OCH}_{2} \mathrm{CF}_{3}\right)_{3}\right)$ and $\mathrm{Os}_{3}(\mathrm{CO})_{9}\left(\mathrm{PPh}_{3}\right)_{3}$. J. Organomet. Chem. 1988, 347, 207-235. 